\title{
Acknowledgement of Reviewers 2021
}

\section{George Tsakiris ${ }^{1}$}

Published online: 9 February 2022

(ᄋ) The Author(s), under exclusive licence to Springer Nature B.V. 2022

Please join us in thanking all those scientists and experts in the various fields represented in Water Resources Management for devoting time and effort to review the papers that we have been sending them. The Editor-in-Chief, the editorial board and publisher acknowledge the colleagues listed below for their excellent reviews of papers for which final decisions have been made during the period 1 January 2021 to 31 December 2021.

Aminuddin Ab Ghani
Ali Abbasi
Mostafa Abdelbaky
Fahmy Abdelhaleem
Noha Abdel-Mottaleb
Fadi Abdelradi
Saipol Bari Abd-karim
Maryam Abdolahzadeh
Isam Abdulhameed
Rajesh Abhay
Sameh Abou Rafee
Ahmed Abou-Shady
João Abrantes
Muhammad Ilyas Abro
Girma Adane
Shahzada Adnan
Ali Afruzi
Zahra Afzali-Gorouh
Pouya Aghelpour
V. Agilan
Ummar Ahad
Ali Ahani
Ishtiyaq Ahmad
Saima Ahmad
Ferdous Ahmed
Xueshan Ai

Zhipin Ai
Huo Aidi
Ahzegbobor Aizebeokhai
Hüseyin Akay
Saeid Akbarifard
Naseem Akhtar
Muhammed Ernur Akıner
Muhanned Al Murib
Ali Al Rammahi
Angelos Alamanos
Yousif Al-Aqeeli
Ahmed Al-Areeq
Sadık Alashan
Seyedhassan Alavinia
Mohammad Albaji
Valeria Alban Dominguez
Selcuk Albut
Ihab Alfadhel
Faisal Alfaisal
Abdelrahman Ali
Andesikuteb Ali
Emad Ali
Imran Ali
Zulfiqar Ali
Farhad Alizadeh
Mohamad Javad Alizadeh

Jafar Al-Jawad
Loai Aljerf
Ahmed Aljuaidi
Mahmoud Al-Khafaji
Sohaib Al-Mamoori
Mustafa Al-Mukhtar
Abdulaziz I. Almulhim
Emran Alotaibi
Shayma Al-Rubaye
Mohanned Al-Sheriadeh
Radwan Al-Weshah
Gholamreza Andalib
Alexandre Andrade
Yasmeen Anis
Libor Ansorge
Mohammad Arab Amiri
Jorge Arevalo
Vicky Ariyanti
Aman Arora
Mohit Arora
Arfan Arshad
Vasan Arunachalam
Mohsen Asadi
Morteza Asadi
Antonia Asencio
Muhammad Ashraf

George Tsakiris gtsakir@central.ntua.gr

1 Lab of Reclamation Works and Water Resources Management, National Technical University, Athens, Greece 
Seyed Mohammad Ashrafi

Hasnat Aslam

Diego Avesani

Leandro Avila

Alex Avilés

Taymoor Awchi

Murat Ay

Alper Aydemir

Francisco Ayuga

Armin Azad

Hazi Azamathulla

Hazi Mohammad Azamathulla

Arash Azari

Asghar Azizian

Monte Azmi

Venkatesh B.

Poojashree B.P.

K.S. Jinesh Babu

Ali Bagheri

Farhad Bahmanpouri

Tao Bai

Bahram Bakhtiari

Adithya Bandari

Reza Barati

Emanuele Barca

Kenia Barros

Mirjana Bartula

Muhammad Basharat

Seyyed Nasser Bashi-Azghadi

Saeed Bazgeer

Neslihan Beden

Behrang Beiranvand

Loucif Benahmed

Holger Benavides-Muñoz

Mohamed Ben-Daoud

Hassan Benfetta

Semra Benzer

Marco Berardi

Saulo Bezerra

Aditi Bhadra

Seemanta Bhagabati

Nirman Bhagat

R. S. Bhalla

Khursheed Bhat

Sajjad haider Bhatti

Sandra Bianucci
Seyed Mostafa Biazar

Omer Bilhan

Prashant Birbal

Biplab Biswas

Frederick Bloetscher

Rewa Bochare

Paweł Bogawski

Sriman Pankaj Boindala

Muhammad Waseem Boota

Petre Bretcan

Kaique Brito Silva

Abebe Tadesse Bulti

Naci Büyükkaracığan

Hi-ryong Byun

Singh C.D.

Jialiang Cai

Yi Cai

Xiatong Cai

Ana Paula Camelo

Marcus André Campos

Laura Canno Ferreira Fais

Shengle Cao

Silvia Carpitella

Ana Claudia Pereira Carvalho

Ana Paula Carvalho

Andreza Carvalho

Stefano Casadei

Carme Castaner

Pedro Wirley Castro Fori

Daysy Cavalcanti

Roberto Cecílio

Gokmen Ceribasi

Cem Cetinkaya

Praveenkumar $\mathrm{Ch}$

Cristian Chadwick

Jacob Chandapillai

Xuexiang Chang

Winai Chaowiwat

Barkha Chaplot

Nikos Charizopoulos

K. W. Chau

Kwok-wing Chau

Duan Chen

Huawei Chen

Jia Chen

Pan Chen
Shu Chen

Wei Chen

Ying-jung Chen

Yizhong Chen

Yuanfang Chen

Hongguang Cheng

Jilin Cheng

Qingping Cheng

Rim Cherif

Po-kuan Chiang

Pascale Chiron

Namjeong Choi

Bahram Choubin

Mustafa

Shahadat Chowdhury

Vasileios Christelis

Shipeng Chu

Mesut Cimen

Kristan Cockerill

Marcelo

Theodora Cohen Liechti

Virginia Rosa Coletta

Nicolò Colombani

Thibaut Cuvelier

Mônica Da Hora

Ismail Dabanli

Chao Dai

Huseyin Dalkilic

Sathish Kumar Damodaran

Ali Danandeh Mehr

Rasoul Daneshfaraz

Chiheng Dang

Tanveer Dar

Bappa Das

Jew Das

Lassâad Dassi

José De Araújo

J.J. De Felipe

Omar Antonio De la Cruz Courtois

Luca De Oliveira Turci

Rafael De Paes

Francesco De Paola

Leydiana De Sousa Pereira

Tiago De Vargas

Surajit Deb Barma

Bayshakhi Deb Nath 


\begin{tabular}{|c|c|c|}
\hline Mridusmita Debnath & Elias Farah & Michele Greco \\
\hline Benjamin Wullobayi Dekongmen & Farshad Farahbod & Jinjin $\mathrm{Gu}$ \\
\hline Roberto Del Teso March & Nima Fayaz & Qing Gu \\
\hline Tamene Adugna Demissie & Atabak Feizi & Mustafa \\
\hline Kamalini Devi & Jingjie Feng & Veysel Gumus \\
\hline Suhas Dhande & Yu-xi Feng & Mert Gunacti \\
\hline Latifa Dhaouadi & João Fernandes & Manoj Gundalia \\
\hline Danyang Di & Francisco Fernández & Fei Guo \\
\hline Vittorio Di Federico & Danieli Ferreira & Lishuo Guo \\
\hline Slava Dineva & Lachezar Filchev & Yuxue Guo \\
\hline Victor Emanuel Diniz & Ehsan Foroumandi & Aditya Gupta \\
\hline António & Tedlaye Forsido & Pankaj Gupta \\
\hline Soumyashree Dixit & Alberto Franco & Sushindra Gupta \\
\hline Alireza Docheshmeh Gorgij & Yicheng Fu & Sunil Gurrapu \\
\hline Funda Dökmen & Mohamed Gabr & Sezgin Hacısalihoĝlu \\
\hline Souag-Gamane Doudja & Hamza Gabriel & Zahreddine Hafsi \\
\hline Pengfei Du & Mohamed Gad & Mohammed Hajeeh \\
\hline Shuping Du & Hadi Galavi & Wiktor Halecki \\
\hline Anja Du Plessis & Armando Gallo Yahn Filho & Mohammed Hamed \\
\hline Kai Duan & Imen Gam & Abdelkader Hamlat \\
\hline Huan-Feng Duan & Xiang Gao & M. Hafedh Hamza \\
\hline Timketa Duguma & Junior Garcia & Songjun Han \\
\hline Rebecca Dziedzic & Marcos García-López & Yongming Han \\
\hline Naser Ebadati & Vaibhav Garg & Md. Hasanuzzaman \\
\hline Elham Ebrahimi & Srishti Gaur & Seied Mehdy Hashemy Shahdany \\
\hline Mihai Eftimie & Syed Geelani & Waqed Hassan \\
\hline Davidson Egirani & Caspar Geelen & Mohammad Reza Hassanvand \\
\hline Alpaslan Ekdal & Brook Getahun & Yanhu He \\
\hline Daniel El Chami & Amir Ghaderi & Shaokun \\
\hline Mena Elassal & Roghayeh Ghasempour & Zhongzheng He \\
\hline Sermin Elevli & Nesrine Ghouili & Salim Heddam \\
\hline Amro Elfeki & Margaret Gitau & Younggu \\
\hline Mohamed Elhag & Carlo Giudicianni & Mathew Herrnegger \\
\hline Hosam Elhegazy & Habtamu Gizaw & Mohammad Heydari \\
\hline Mohamed El-Mahdy & Patricia Gober & Mirjana Horvat \\
\hline Abdelkader Elouissi & Marcos Henrique Godoi & Zoltan Horvat \\
\hline Mustafa El-Rawy & Mohammad Hossein Golmohammadi & Farzad Hosseini Hossein Abadi \\
\hline Hassan Emami & Ricardo Gomes & Seyed-Mohammad Hosseini-Moghari \\
\hline Hamit Erdal & Dennis Gonzalez & Zeinab Hosseinzadehghazichaki \\
\hline Marina Erechtchoukova & Ana Cristina González Valoys & Jingming Hou \\
\hline Daniel Eshete & Rebeca González-Villela & Eva Audrey Yessito Houessou-Dossou \\
\hline Maria Esteller & Girish Gopinath & Chunming $\mathrm{Hu}$ \\
\hline Rasha Fadhil & J. Götzinger & $\mathrm{Hu} \mathrm{Hu}$ \\
\hline Md Golam Rabbani Fahad & Chandre Gowda C. & Mianhao $\mathrm{Hu}$ \\
\hline Fernando Fan & Manish Kumar Goyal & Zhigen $\mathrm{Hu}$ \\
\hline Liangxin Fan & Francesco Granata & Cheng-Chia Huang \\
\hline
\end{tabular}




\begin{tabular}{|c|c|}
\hline Daohan Huang & Suresh Khandelwal \\
\hline Xuan Huang & Roghayeh Khosravi Savadjani \\
\hline Ijaz Hussain & Tae-woong Kim \\
\hline Rana Muhammad Adnan Ikram & Yong Gun Kim \\
\hline Vijiraja Ilayarajan & Sanjeev Kimothi \\
\hline Aleksandra Ilić & Umut Kırdemir \\
\hline Shashi Indwar & Wesley Kirui \\
\hline Ahmet Irvem & Ozgur Kisi \\
\hline Md Monirul Islam & Burak Kızılöz \\
\hline Ardalan Izadi & Kerim Koc \\
\hline Mehran Jafari & Serdar Korkmaz \\
\hline Ahmad Jafarzadeh & Cihangir Koycegiz \\
\hline Hamidreza Jahangirzadeh & Anjala Krishen \\
\hline Priyanshu Jain & Ashok Kumar \\
\hline Rahul Jaiswal & Dilip Kumar \\
\hline Ali Akbar Jamali & Vijendra Kumar \\
\hline Piyapong Janmaimool & Mogaraju Jagadish Kumar \\
\hline Saman Javadi & Pavan Kumar Kummamuru \\
\hline Ponselvi Jeevaragagam & Alban Kuriqi \\
\hline Joygopal Jena & Volker Lämmchen \\
\hline Zhonghua Jia & Feng Lan \\
\hline Liu Jiaming & Sarmad Dashti Latif \\
\hline Nura Jilo & William Laton \\
\hline Lei Jin & Agostino Lauria \\
\hline Naveen Joseph & Doron Lavee \\
\hline Mehdi Jovzi & Do-hun Lee \\
\hline Bongseog Jung & Zhang Letao \\
\hline Ali K.Ghorbanpour & Ear Lewis \\
\hline Pradip Kalbar & Changbin Li \\
\hline Ren Kang & Fan $\mathrm{Li}$ \\
\hline Shamsa Kanwal & Fawen $\mathrm{Li}$ \\
\hline Atilla Karataş & Hang Li \\
\hline Hamid Kardan Moghaddam & Lei Li \\
\hline Neamat Karimi & Xungui Li \\
\hline Keivan Karimlou & Zhiwei Li \\
\hline Akmal Karimov & Bin Liu \\
\hline Zahra Kashkaki & Suning Liu \\
\hline Norhafezah Kasmuri & Gang Liu \\
\hline Seetharam Katakam & Zhao Liu \\
\hline Yashwant Katpatal & Abbas Ljam \\
\hline Mulu Kerebih & Athanasios Loukas \\
\hline Hamed Ketabchi & Zhixiang Lu \\
\hline Mohammad Javad Keyhanpour & Bin Luo \\
\hline Mohammad Reza Khaleghi & Pingping Luo \\
\hline Kamran Khalifehei & Yun Luo \\
\hline Noor Khan & Janga Ready M \\
\hline
\end{tabular}

U. Maalik

Mohamad Reza Madadi

Ulises Rodrigo Magdalena

Mahmoud Maghrebi

Ali Mahaqi

Omar Mahmood Agha

Mohammad Mahmoudi-Rad

Bahram Malekmohammadi

Anurag Malik

Uday Mandal

Joyce Maphanyane

Milica Markovic

Richarde Marques Da Silva

Herlander Mata-Lima

Gagan Matta

Joana Medeiros

Darshan Mehta

Shein Mezour

Numan Mizyed

Ivana Mladenović-Ranisavljević

Firdaus Mohamad Hamzah

Mahmoud Mohammad Rezapour Tabari

Maryam Mohammadpour

Ruqayah Mohammed

Abinash Mohanta

Ali Mohtashami

Jose-Luis Molina

Hamed Moradi

Sayed Masood Mostafavi Darani

S. Hooman Mousavi

B. Msh

Tianwei Mu

Noryanti Muhammad

Christos Myriounis

Dimitrios Myronidis

Ramsundram N.

Ali Reza Nafarzadegan

Amirhossein Najafabadipour

I. Nalbantis

Ramakrishna Nallathiga

Umamahesh Nanduri

Daniel Nascimento-E-Silva

Mohammad Nazeri Tahroudi

Thuy Ngo

Xiaokuan Ni 


\begin{tabular}{|c|c|c|}
\hline Xizhi Nong & Bebitta R. & Rahaf Shakko \\
\hline M. Masud Noruzi & Majeed Ramal & Farzaneh Shams \\
\hline Eric Noubissié & Venkata Ramana Rambha & Mahmoud Shams \\
\hline Vahid Nourani & Yousef Ramezani & Nagarajan Shanmugavel \\
\hline Ines Nsiri & Rezan Rasheed & Durga Sharma \\
\hline Taís Maria Nunes Carvalho & Eeswaran Rasu & Prabhakar Sharma \\
\hline Izuchukwu Obiadi & Ali Raza & Suresh Sharma \\
\hline Enovwo & Suresh Reddy & Uttam Sharma \\
\hline Padam Omar & Ajaybhaskar Reddy & Kamran Shayesteh \\
\hline Mohammed Omer & Inga Retike & Muhammad Shehzad \\
\hline Jose Pablo Ortiz Partida & Abdul Razaq Rezaei & Farooq Sher \\
\hline Kaveh Ostad-Ali-Askari & Hossien Riahi Madvar & Haoran Shi \\
\hline Mehmet Ozger & Jose Ribas & Jenq-tzong Shiau \\
\hline Ismail Özölçer & Sultan Rizvi & David ching-fang Shih \\
\hline Sudheer Padikkal & Anna Robak & Mohammad Shooshtarian \\
\hline Roberta Padulano & Joao Rocha & Mojtaba \\
\hline Alessandro Pagano & Gonçalo Rodrigues & Jorge Silva \\
\hline Magdalena Pajak Subodh Pal & Abbas Roozbahani & Pedro Silva \\
\hline Puneeta Pandey & Amir Roshan & Wesley Silva \\
\hline Sandeep Pandey & Bouchrit Rouissat & Gurjeet Singh \\
\hline Hari Pant & Mohsen Saadat & Shekhar Singh \\
\hline Daeryong Park & Amin Sadeqi & Leelambar Singh \\
\hline Antônio Pasqualetto & Fouad Saeed & Prateek Kumar Singh \\
\hline Anilkumar Pathare & Mohammadreza Safaei & Ajit Pratap Singh \\
\hline Roya Peirovi & Abhisekh Saha & Anupam \\
\hline Filipe da Silva Peixoto & Probal Saha & Swati Sirsant \\
\hline Luis Pereira & Satyabrata Saha & V. Sivakumar \\
\hline Agustí Pérez-Foguet & Saurav Saha & Adebayo Sojobi \\
\hline Miguel Angel Pérez-Martín & Meysam Salarijazi & Jaysukh Songara \\
\hline Sabreen Pibars & Luiz Felippe Salemi & Shan-e-Hyder Soomro \\
\hline Izabela Piegdoń & Amir Hossein Salimi & Ioannis Souliotis \\
\hline Katarzyna Pietrucha-Urbanik & Abrari Salleh & Bojan Srdjevic \\
\hline Cicero Pimenteira & Mahsa Samadi-Darafshani & K. Srinivasa Raju \\
\hline Ahmadreza Pirali Zefrehei & Francisco-Javier Sánchez-Romero & Justyna Stańczyk \\
\hline Rudra Mohan Pradhan & Luis Sañudo-Fontaneda & Zoran Štirbanović \\
\hline M.N. Prakash & Anindita Sarkar & Qingmu Su \\
\hline Virendra Proag & Jerusa Schneider & Fuzieah Subari \\
\hline Mario Procopiuck & Zekâi Şen & Jueyi Sui \\
\hline Francesco Pugliese & Alfonso Senatore & Mohd Sofiyan Sulaiman \\
\hline Peng Qi & Arsalan Sepehri & Sadeq Oleiwi Sulaiman \\
\hline Changhai Qin & Pelin Sertyeşilişık & Ali H. Ahmed Suliman \\
\hline Huanhuan Qin & Rulli Pratiwi Setiawan & Qamar Sultana \\
\hline Emanuele Quaranta & Sadra Shadkani & Feifei Sun \\
\hline Majid Rahimpour & Hosna Shafaei & Feng Sun \\
\hline Mohammad Mahdi Rajabi & Muhammad Shahid & Fengyun Sun \\
\hline Pradeep Rajput & Abdol Aziz Shahraki & Hongguang Sun \\
\hline
\end{tabular}




\begin{tabular}{|c|c|c|}
\hline Xinguo Sun & Liang Wang & Xiaofang Yue \\
\hline Rama T. Rashad & Peng Wang & Javad Zahiri \\
\hline Hiroyuki Takeshima & Shun-Jiu Wang & Mohammad Zakwan \\
\hline Haoqiang Tan & Tian Wang & Abdol Rassoul Zarei \\
\hline Xianzhe Tang & Wenbing Wang & Mohanna Zarei \\
\hline Zhonghua Tang & Xiaoping Wang & Martina Zelenakova \\
\hline Ahad Hasan Tanim & Yongtao Wang & Abhijit Zende \\
\hline Tiku Tanyimboh & Yuhao Wang & Xiang Zeng \\
\hline Hasan Tatli & Yuqiu Wang & Yebegaeshet Zerihun \\
\hline Guilherme Teixeira & Julio Wasserman & Nejat Zeydalinejad \\
\hline Telma Teixeira & Ting Wei & Luxin Zhai \\
\hline Sanju Thomas & Tianfu Wen & Xiaoyan Zhai \\
\hline Dimitris Tigkas & Xiaohu Wen & Chen Zhang \\
\hline Nity Tirkey & Xin Wen & Fan Zhang \\
\hline Arvind Tomar & Yong Jie Wong & Jin Zhang \\
\hline Juan Antonio Torres-Martínez & Endashaw Workie & Mengjie Zhang \\
\hline Gaurav Tripathi & Dan Wu & Min Zhang \\
\hline Mahesh Tripathi & Guanghong Wu & Renlong Zhang \\
\hline Mahesh Tripathi & Penghai Wu & Rui Zhang \\
\hline Flavio Trojan & Xilong Wu & Ruilian Zhang \\
\hline Demetrios Tsesmelis & Yanfeng Wu & Xiaoli Zhang \\
\hline Nurul Nadrah Aqilah Tukimat & Yanqing Wu & Yanhong Zhang \\
\hline Yunfei Tuo & $\mathrm{Zh} \mathrm{Wu}$ & Qiang Zhao \\
\hline Mustafa Turan & Nan Xiang & Tony Zhao \\
\hline Veysel Turan & Yang Xiao & Gao Zhendong \\
\hline Evren Turhan & Zhangling Xiao & Tianyuan Zheng \\
\hline Tuvia Turkeltaub & Yu jiu Xiong & Ming Zhong \\
\hline Bulent Tutmez & Guanglai Xu & Ping-an Zhong \\
\hline Duygu Ulker & Shengqing Xu & Shuai Zhong \\
\hline Myoung-jin Um & Xuan Xu & Jingnan Zhou \\
\hline Koichi Unami & Zhengguang Xu & Qiuwen Zhou \\
\hline Pawan Upadhyay & Jiuping Xu & Shenbei Zhou \\
\hline Kumar V. & Mehdi Yaltaghian Khiabani & Zhigao Zhou \\
\hline Babak Vaheddoost & Omid A. Yamini & Bin Zhu \\
\hline Mahdi Valikhan Anaraki & Lei Yan & Liangjun Zhu \\
\hline Tom Van Der Voorn & Walter Yana-De La Riva & Yilin Zhuang \\
\hline Ajay Kumar Vashisht & Lihu Yang & Elvis Žic \\
\hline Rambha Venkataramana & Qingchun Yang & Alexandros Ziogas \\
\hline Giacomo Viccione & Yang Yang & Mehdi Zomorodian \\
\hline Vikas Kumar Vidyarthi & Alpaslan Yarar & Mohammad Zounemat-Kermani \\
\hline Gonzalo Villa-Cox & Zaher Yaseen & Tomasz Zubala \\
\hline María C. Villarín & Tsuyoshi Yoda & \\
\hline Zisis Vryzas & Xueyi You & \\
\hline Wuyi Wan & Huan Yu & \\
\hline Bohong Wang & Jing Yu & \\
\hline Fei Wang & Xue Yu & \\
\hline Hongrui Wang & Meihua Yuan & \\
\hline
\end{tabular}

We apologize for any errors or inadvertent omission.

Publisher's Note Springer Nature remains neutral with regard to jurisdictional claims in published maps and institutional affiliations. 